\title{
Anti-dentine Salivary SIgA in young adults with a history of dental trauma in deciduous teeth
}

\section{Gabriela Fleury SEIXAS(a) Cássia Cilene Dezan GARBELINI(a) Kelly Limi AIDA ${ }^{(a)}$ \\ Ana Paula CHEIRUBIM(b) \\ Emerson José VENÂNCIO(b) \\ Solange de Paula RAMOS(b)}

\footnotetext{
(a) Universidade Estadual de Londrina - UEL, School of Dentistry, Center of Health

Sciences, Londrina, PR, Brazil.

(b) Universidade Estadual de Londrina - UEL, School of Dentistry, Center of Biological Sciences, Londrina, PR, Brazil.
}

Declaration of Interests: The authors certify that they have no commercial or associative interest that represents a conflict of interest in connection with the manuscript.

Corresponding Author:

Gabriela Fleury Seixas

E-mail: fleuryseixas@gmail.com

DOI: 10.1590/1807-3107BOR-2015.vol29.0102

Submitted: Aug 30, 2014

Accepted for publication: Apr 16, 2015

Last revision: Jul 07, 2015

\begin{abstract}
Anti-dentin autoantibodies are associated with inflammatory root resorption in permanent teeth and are modulated by dental trauma and orthodontic force. However, it is not known whether deciduous tooth trauma can stimulate the development of a humoral immune response against dentin. The aim of this study was to evaluate the levels of salivary SIgA reactivity against human dentin extract in young adults with a history of trauma in the primary dentition. A sample of 78 patients, aged 18 to 25 , who had completed an early childhood ( 0 to 5 years old) caries prevention program years earlier at the Universidade Estadual de Londrina Pediatric Clinic, underwent radiographic examination and salivary sampling. Anti-dentin SIgA levels were analyzed by immunoenzymatic assay and Western blotting. Although dental trauma to deciduous teeth had occurred in $34(43.6 \%)$ of the patients, no differences in SIgA levels were detected between individuals who had experienced trauma and those who had not $(p>0.05)$. Multivariate regression analysis showed no association between dental trauma and SIgA levels ( $p>0.05$ ). Patients with a history of deciduous trauma presented low levels of anti-dentin antibodies, associated with orthodontic root resorption $(p<0.05)$. Western blot analysis showed that salivary antibodies recognized a single band of approximately $45 \mathrm{kDa}$ in dentin extract. We concluded that salivary SIgA recognizes a specific component of the dentin matrix and that anti-dentin antibodies were not triggered by trauma to primary teeth. However, trauma to deciduous teeth may down-modulate SIgA in response to orthodontic root response.
\end{abstract}

Keywords: Dentin; Tooth Resorption; Autoimmunity; Dental Care for Children; Saliva.

\section{Introduction}

Dental trauma in deciduous teeth is highly prevalent in pediatric clinics and can involve sequelae and complications in both deciduous and permanent dentition. Premature root resorption of primary teeth is one of the main complications and may lead to the untimely loss of traumatized teeth. ${ }^{1,2}$ Bille et al. ${ }^{3}$ also suggested that premature root resorption of primary teeth may be associated with an increased risk of root resorption of permanent teeth. 
The biological mechanism of pathological root resorption has not yet been completely elucidated, and individual susceptibility to root resorption may be associated with an autoimmune response to the dentin matrix. ${ }^{4,5,6,7}$ Differences in anti-dentin antibody levels have been found in experimentally induced root resorption in rodents ${ }^{4,5}$ and in patients with root resorption after dental trauma to permanent teeth or orthodontically induced root resorption. ${ }^{6,7}$ High levels of anti-dentin antibodies were found in patients with root resorption due to dental trauma in permanent teeth, ${ }^{6}$ as well as in patients with an increased risk to orthodontic root resorption. ${ }^{7}$ These results suggest that a specific immune response to dentin antigens may play a role in the root resorption of permanent teeth. Indeed, effector mechanisms of the immune response and autoimmunity have been described during resorption of mineralized tissues such as bone matrix. ${ }^{8}$

It has been suggested that dental trauma to the permanent dentition favors the development of an immune response against dentin components ${ }^{6}$ and is associated with root resorption. ${ }^{6,7}$ However, the impact of dental trauma to deciduous teeth on the development of this immune response or on the root resorption of permanent teeth is not known. Trauma to primary teeth occurs frequently in children up to 24 months of age. ${ }^{2,9,10}$ Innate and adaptive immune systems are immature in newborns and tend toward a humoral immune response and the recognition of protein antigens. ${ }^{11,12,13,14}$ The presence of a local chronic inflammatory reaction associated with the immaturity of a child's immune system may favor the recognition of self-antigens, thus increasing the risk of autoimmune disorders. ${ }^{15,16}$ Serum and salivary autoantibodies against dentin antigens have been found in patients with replacement resorption and orthodontically induced root resorption. ${ }^{6,7}$ The origin and role of these autoantibodies are not known. In relation to dental trauma, the exposure of dentin in an inflammatory milieu may prime antigen-presenting cells to trigger a local immune reaction prompted especially by salivary autoantibodies against the dentin matrix.

Considering the high prevalence of dental trauma in children, whose immune systems are maturing, and the influence of dentin autoimmunity in pathological root resorption, the objective of this study was to investigate anti-dentin salivary IgA (SIgA) levels in young adults who suffered dental trauma to primary teeth.

\section{Methodology Subjects}

All procedures were performed after the volunteers had signed an informed consent previously approved by the Research Ethics Committee for Human Experiments of the Universidade Estadual de Londrina - UEL, protocol 209/2011.

This study involved a retrospective cohort of 18-to-25-year-olds who had mandatorily completed an early childhood caries prevention program years earlier at 0 to 5 years of age, at UEL's Pediatric Clinic. Patient recruitment was based on the records of the Pediatric Clinic from 1986 to 1994. Prospective participants who had been using steroidal or non-steroidal anti-inflammatory drugs for the two previous months and/or continuous use of cholinergic or adrenergic drugs, or who presented chronic inflammatory diseases or oral mucosal lesions were excluded. Patients undergoing orthodontic treatment were also excluded.

Data on trauma to deciduous teeth, severity of the trauma, recurrent traumatic episodes and lesion evolution were collected directly from the patients' records. Trauma was classified by frequency, as either a single episode or recurrent episodes, and by severity, as either simple (subluxation, enamel or enamel dentin fractures without pulp exposure) or complicated (lateral luxation, intrusion, extrusion, avulsion or coronary fractures with pulp exposure). Information on trauma to permanent teeth, previous orthodontic treatment, medical history and oral health was collected during anamnesis and an oral inspection.

Unstimulated whole saliva samples $(2 \mathrm{ml})$ were collected by expectoration into sterilized vials after the subjects had rinsed their mouths twice with deionized water. Saliva samples were centrifuged at $12,000 \mathrm{rpm}$ for 10 minutes, and the supernatants were then stored at $-20^{\circ} \mathrm{C}$ until use. The saliva secretion rate was calculated according to the volume of saliva 
secreted per minute. The SIgA secretion rate was calculated according to the amount of SIgA secreted per minute.

Radiographs $(70 \mathrm{kV}, 10 \mathrm{~mA}$, and exposure time of 0.7 seconds) of the upper central and lateral incisors were taken using the long-cone paralleling technique. These teeth were radiographed because of the high prevalence of orthodontic root resorption. In our study, patients reported dental trauma of permanent teeth only in upper incisors. Two independent, trained and blinded examiners evaluated each radiograph. The kappa coefficient shows 95\% agreement between examiners. Root resorption was classified as either present or absent. In cases of inter-observer disagreement, consensus was reached by discussion.

\section{Antigen preparation}

Human dentin extract (HDE), a crude extract containing the organic material of the dentin matrix, was used as the antigen. HDE was obtained using a modified version of Wheeler and Stroup ${ }^{5}$ technique, and involved donated third molars whose extraction had been previously recommended. Protein concentration (ranging from 300 to $400 \mathrm{mg} / \mathrm{ml}$ ) was determined by the Bradford method, using bovine serum albumin as a control. Two third molars were used to extract $1.05 \mathrm{mg} \cdot \mathrm{ml}^{-1} \mathrm{HDE}$. The antigen was stored at $-20^{\circ} \mathrm{C}$ until use.

\section{Immunoenzymatic assay to detect anti-dentin salivary IgA}

$\mathrm{HDE}$ (300 $\mathrm{mg}$ protein $/ \mathrm{ml}$ ) in carbonate-bicarbonate buffer $\left(\mathrm{Na}_{2} \mathrm{CO}_{3} 1.59 \mathrm{~g}, \mathrm{NaHCO}_{3} 2.93 \mathrm{~g}\right.$, distilled water qsp $1000 \mathrm{ml}, \mathrm{pH}$ 9.6) was used to coat 96-well immuno plates (Techno Plastic Products, Zurich, Switzerland) for 1 hour at $37^{\circ} \mathrm{C}$, after which they were stored overnight at $4^{\circ} \mathrm{C}$. The plates were washed four times with PBS containing 0.05\% Tween 20 (PBST) blocked with PBS-T-5\% skim milk for 1 hour at room temperature. After the plates were washed, the saliva samples $(100 \mu \mathrm{l})$ were incubated at $37^{\circ} \mathrm{C}$ for 2 hours, washed four times and incubated with anti-human IgA labeled with peroxidase antibody (A88-102P, Bethyl Laboratories, Montgomery, USA), and diluted (1:50.000), at $37^{\circ} \mathrm{C}$ for 1 hour. After washing the test plates, $100 \mu \mathrm{l}$ of the tetramethylbenzidine substrate solution was added. After 15 minutes, the reaction was stopped with $50 \mu \mathrm{H}_{2} \mathrm{SO}_{4} 4 \mathrm{~N}$, and the absorbance was read in a Multiskan EX reader (Lab Systems, Helsinki, Finland) at $450 \mathrm{~nm}$. Antibody levels were expressed according to absorbance in optical density (OD) units.

\section{Western blot analysis}

HDE (10 or $20 \mu \mathrm{g} \cdot \mathrm{ml}^{-1}$ ) was diluted in sample buffer $(62.5 \mathrm{mmol} / \mathrm{L}$ Tris-HCl, $2 \%$ SDS, $10 \%$ glycerol, $5 \% \beta$-mercaptoethanol) and heated for $5 \mathrm{~min}$ at $90^{\circ} \mathrm{C}$ to perform the SDS-PAGE with $10 \%$ acrylamide/bis-acrylamide gels. The electrophoresis gels were stained with Coomassie Blue or silver nitrate impregnation. Proteins $\left(10 \mu \mathrm{g} . \mathrm{ml}^{-1}\right)$ were transferred electrophoretically to polyvinylidene difluoride (PVDF) membranes. PVDF membranes were blocked with $5 \%$ low-fat dried milk powder, dissolved in Tris buffer saline containing $0.1 \%$ Tween 20 (TBS-T) for 2 hours at room temperature. The membranes were washed in PBS and incubated with undiluted saliva samples for 2 hours at room temperature. Finally, the membranes were incubated with anti-human IgA antibody conjugated to peroxidase (A88-102P, Bethyl Laboratories, Montgomery, USA), diluted at 1:10000, for 1 hour at room temperature. Immunoreactive bands were revealed using a diaminobenzidine solution.

\section{Statistical analysis}

The Kolmogorov-Smirnov test was used to test normality, and both parametric tests (Student's $t$-test, ANOVA/Tukey) and non-parametric tests (Mann-Whitney and Kruskal-Wallis/Dunn's tests) were applied to detect differences among study variables. Spearman's rank correlation coefficient was used to detect correlations between SIgA levels and age of trauma onset. Multivariate regression analysis was performed with the presence of root resorption in permanent teeth as the outcome variable. $p$-values less than 0.05 were considered statistically significant.

\section{Results}

A total of 559 clinical records were located for the 0-to-5-year-old patients who had received treatment and completed the Pediatric Clinic's dental caries prevention program between January 1986 and 
December 1998. Of the 245 (43.8\%) patients who were found and invited to participate in the study, 78 $(13.9 \%)$-all of whom underwent clinical evaluation at UEL's University Dental Clinic - were included in the study.

Of this number, $37(47.4 \%)$ were male and $41(52.7 \%)$ were female. Their mean age was $20.4 \pm 1.7$ years old. Thirty-seven $(47.4 \%)$ had undergone orthodontic treatment and 9 (11.5\%) reported trauma to permanent dentition.

Regarding trauma to deciduous teeth, 34 (43.6\%) volunteers had suffered dental trauma in one or more deciduous incisors. The mean age of trauma to deciduous teeth was $33.4 \pm 16.4$ months of age.

The median level of anti-dentin salivary SIgA was 0.355 (0.270-0.442, 25\%-75\% interquartile) with an optical density (OD) of $450 \mathrm{~nm}$. No differences were observed between individuals with or without deciduous trauma (Figure 1). Recurrent deciduous trauma $(0.352 \pm 0.05 \mathrm{OD})$ did not alter anti-dentin SIgA levels, as compared with single episode trauma $(0.374 \pm 0.12 \mathrm{OD} ; \mathrm{p}>0.05)$. No significant differences in mean SIgA levels were observed between complicated trauma $(0.368 \pm 0.127 \mathrm{OD})$ and non-complicated trauma $(0.355 \pm 0.093$ OD).The types of trauma treatment reported in the clinical records were monitoring $(0.347 \pm 0.10$ OD; $p>0.05)$, extraction and pulpectomy (0.431 \pm 0.09 OD; $p>0.05)$; the treatment type did not influence the salivary SIgA level.

No correlation was observed between total antibody level and secretion rate with mean age of deciduous dental trauma (Figure 2).

Multivariate regression analysis showed no association between anti-dentin SIgA levels and allergies, ethnicity, orthodontic treatment or dental trauma in deciduous or permanent teeth (Table 1).

The frequency of root resorption of permanent teeth was higher in patients who had undergone orthodontic


Figure 1. Analysis of (a) salivary SIgA levels (SIgA) and (b) the secretion rate of SIgA in patients who suffered dental trauma to deciduous teeth and non-trauma patients. The bar represents the median value. $p>0.05$, Mann-Whitney $U$ test.


Figure 2. Correlation between (a) anti-dentin SlgA level and (b) secretion rate with age of dental trauma onset. Spearman's correlation test. 
treatment ( $n=20,57.1 \%$; $p<0.001$, Chi-square test with Yates' correction) than in those who had not $(n=8$; $19.5 \%$ ). However, the frequency of orthodontic root resorption was not significantly different between deciduous trauma patients $(n=8 ; 53.3 \%$; $p>0.05$, Fisher exact test) and non-trauma patients $(\mathrm{n}=12$; $60 \%$ ). Non-trauma patients who presented orthodontic root resorption had increased levels of anti-dentin antibodies. However, orthodontic root resorption in trauma patients was associated with low levels of anti-dentin SIgA (Figure 3).

The various components of the HDE extract $(10 \mu \mathrm{g}$ protein $\mu \mathrm{l}^{-1}$ ) had different concentrations and molecular weights. SDS-PAGE showed HDE components with approximate molecular weights of $80,73,65,55,45$, 35, 30, 20 and $15 \mathrm{kDa}$ (Figure 4). The Western blot analysis showed that saliva antibodies recognized a single band of $\sim 45 \mathrm{kDa}$ (Figure 5).

Table 1. Multivariate regression analysis of anti-dentine SIgA secretion rate and study variables.

\begin{tabular}{lcccc}
\hline Variable & Coefficient & Standard error & $F$ test & $P$ \\
\hline Allergy & -0.006 & 0.080 & 0.0060 & 0.93 \\
Ethnicity & -0.011 & 0.013 & 0.7235 & 0.39 \\
Orthodontic treatment & 0.047 & 0.028 & 2.7887 & 0.09 \\
Root resorption & 0.013 & 0.030 & 0.2045 & 0.65 \\
Gender & -0.013 & 0.031 & 0.1656 & 0.68 \\
Trauma to deciduous teeth & 0.003 & 0.032 & 0.0072 & 0.93 \\
Trauma to permanent teeth & -0.002 & 0.044 & 0.0024 & 0.96 \\
Constant & 0.326 & 0.213 & 2.3412 & 0.13 \\
\hline \hline
\end{tabular}

Correlation coefficient: $r^{2}=0.06$.

\section{Discussion}

The main finding of this study was that dental trauma in deciduous teeth may influence anti-dentin salivary SIgA response in young adults under orthodontic treatment, but does not increase resorption frequency in permanent teeth. Curiously, the local immune response against dentin extract seems to be specific to a single antigen with a molecular weight of $\sim 45 \mathrm{kDa}$.

Root resorption of permanent teeth is a highly prevalent and multifactorial event, and is associated with mechanical and biological mechanisms. ${ }^{17,18}$ Some risk factors, such as age, gender, ethnicity, root shape, dental trauma, orthodontic treatment planning, infection, habits, respiratory allergy, autoimmunity and genetic predisposition, have been commonly associated with root resorption., ${ }^{7,17,18,19,20}$ However, multivariate regression analysis did not detect a significant association among anti-dentin SIgA levels, dental trauma in deciduous teeth and other risk factors for root resorption in permanent teeth.

Autoimmunity against dentin antigens may be associated with individual susceptibility to inflammatory root resorption, considering the key role of the immune system in regulating the resorption of calcified tissues. ${ }^{4,7,8}$ This hypothesis is corroborated by clinical studies reporting increased anti-dentin antibody levels in patients with replacement root resorption ${ }^{6}$ and in patients who are susceptible to orthodontic root resorption. ${ }^{7}$ Moreover, experimental studies have observed an association between


Figure 3. (a) Anti-dentin SIgA levels and (b) secretion rates in orthodontically treated patients with or without trauma of deciduous teeth. The white column represents patients with no radiographic signs of root resorption in permanent upper incisors. Black columns represent patients presenting root resorption in permanent upper incisors. Root resorption: ${ }^{* *} p<0.01$; ${ }^{*} p<0.05$, Student's t-test. 


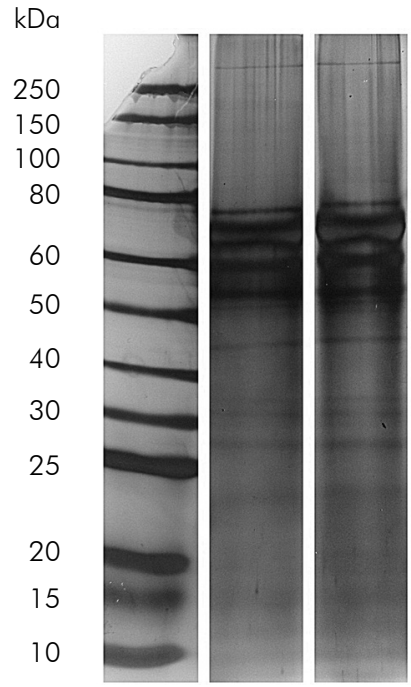

Figure 4. SDS-PAGE of HDE antigen stained with silver nitrate.



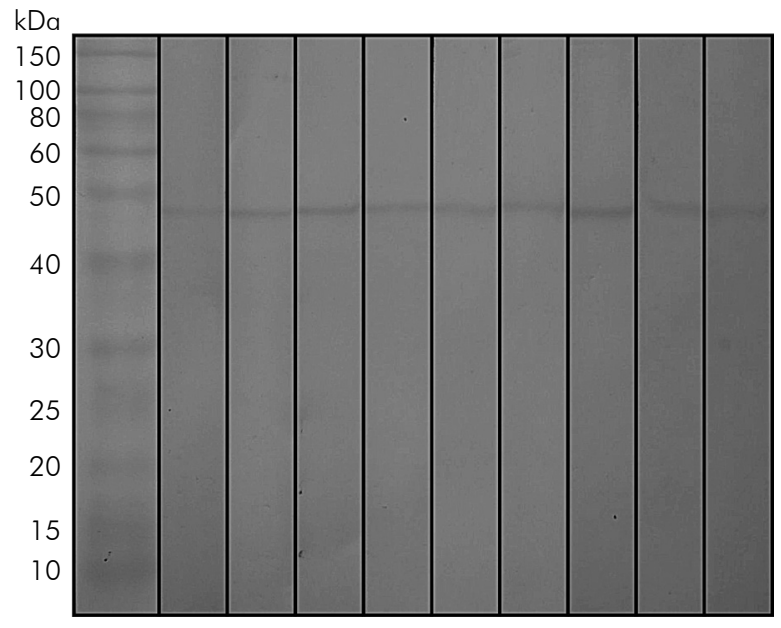

Figure 5. Western blot analysis of saliva samples. Recognition pattern of anti-HDE SIgA in saliva of traumatized and non-traumatized patients. The first column presents molecular weights. Blots 1 to 5 were saliva samples of traumatized patients and blots 6 to 9 , of non-traumatized patients.

trauma-induced root resorption and the presence of anti-dentin antibodies. ${ }^{4,5}$ On the other hand, studies have unanimously observed that antibodies against the dentin matrix are present, even in patients without clinical or radiographic signs of root resorption. ${ }^{6,7}$ The origin of these antibodies is unknown. Our results suggest that dental trauma in primary teeth is not a long-lasting stimulus for increased production of anti-dentin SIgA antibodies. However, trauma to deciduous teeth may predispose individuals to down-modulation of antibody levels and secretion rates in the presence of a local inflammatory event. Caution should be taken when interpreting data about anti-dentin antibody levels and secretion rates of saliva from orthodontically treated patients. The small sample used in this study keeps us from drawing any strong conclusions.

Although it has been demonstrated that trauma to permanent dentition can induce a specific response against dentin antigens, nothing is known about humoral immune response after trauma to deciduous teeth. Dental trauma has a high prevalence rate among preschool children,, 21 and usually leads to quick root resorption and premature exfoliation of traumatized teeth, in children between 18-52 months of age. ${ }^{1}$ Children up to 24 months of age have immature innate and adaptive immune systems that tend toward a humoral response against protein antigens during this period. ${ }^{11,13}$ Our hypothesis was that an inflammatory injury, such as trauma to deciduous teeth, could induce the production of anti-dentin antibodies and memory cells. This hypothesis could not be proven in the present study, since increased levels of anti-dentin antibodies were not found in patients who had suffered deciduous dental trauma. We also found no evidence that trauma to deciduous teeth influences the occurrence of root resorption in adults.

Although trauma to deciduous teeth did not increase anti-dentin SIgA levels in young adults, our results suggest that the humoral immune response may be modulated in the presence of a new inflammatory event in dentin. In agreement with a previous study, increased levels of anti-dentin SIgA were found in patients with orthodontically induced root resorption. ${ }^{7}$ However, low levels of antibody were found in patients who suffered trauma to deciduous teeth and later developed orthodontic root resorption.

We also investigated SIgA recognition of dentin antibodies by Western blot analysis. The HDE used in the present study indicated the presence of several components with molecular weights of $80,73,65,55$, $45,35,30,20$ and $15 \mathrm{kDa}$. Based on the electrophoretic profile described by other authors, the molecules observed in our gel electrophoresis may correspond to known components of the dentin matrix, such as 
matrix Gla protein, bone morphogenetic protein, dentin phosphoprotein, osteonectin, dentin matrix protein 1, osteopontin, extracellular matrix protein and dentin sialophosphoprotein. ${ }^{22,23}$ The Western blot analysis of saliva samples indicated recognition of a single antigen of $\sim 45 \mathrm{kDa}$. According to the molecular weight of dentin matrix components described by Jágr et al., ${ }^{23}$ several molecules, including the precursor of alpha-1 antitrypsin, osteomodulin, calreticulin, alpha enolase, tubulin and secernin-1, have molecular weights near $45 \mathrm{kDa}$. Specific antibodies should be used to identify this specific component. Trauma to deciduous teeth did not induce recognition of other dentin components. This result suggests that SIgA against the $\sim 45 \mathrm{kDa}$ antigen may be produced naturally and may be modulated by inflammatory events in dentin. To this end, further investigation on purified $\sim 45 \mathrm{kDa}$ antigen and its immunogenic potential may

\section{References}

1. Cardoso M, Rocha MJ. Identification of factors associated with pathological root resorption in traumatized primary teeth. Dent Traumatol. 2008 Jun;24(3):343-9.

2. Assuncao LR, Ferelle A, Iwakura ML, Nascimento LS, Cunha RF. Luxation injuries in primary teeth: A retrospective study in children assisted at an emergency service. Braz Oral Res. 2011 Mar-Apr;25(2):150-6.

3. Bille ML, Kvetny MJ, Kjaer I. A possible association between early apical resorption of primary teeth and ectodermal characteristics of the permanent dentition. Eur J Orthod. 2008 Aug;30(4):346-51.

4. Ng KT, King GJ, Courts FJ. Humoral immune response to active root resorption with a murine model. Am J Orthod Dentofacial Orthop. 1990 Nov;98(5):456-62.

5. Wheeler TT, Stroup SE. Traumatic root resorption in dentine-immunized mice. Am J Orthod Dentofacial Orthop. 1993 Apr;103(4):352-7.

6. Hidalgo MM, Itano EN, Consolaro A. Humoral immune response of patients with dental trauma and consequent replacement resorption. Dent Traumatol. 2005 Aug;21(4):218-21.

7. Ramos SP, Ortolan GO, Dos Santos LM, Tobouti PL, Hidalgo MM, Consolaro A, Itano EN. Anti-dentine antibodies with root resorption during orthodontic treatment. Eur J Orthod. 2011 Oct;33(5):584-91.

8. Takayanagi H. Osteoimmunology: Shared mechanisms and crosstalk between the immune and bone systems. Nat Rev Immunol. 2007 Apr;7(4):292-304. help us understand the role of autoantibodies against dentin in inflammatory root resorption.

\section{Conclusions}

Anti-dentin salivary SIgA recognizes a $\sim 45 \mathrm{kDa}$ antigen present in the dentin matrix. The presence of these antibodies was not triggered by trauma to the primary dentition, but could possibly be modulated by inflammatory events on the dentin surface. Our results suggest that dental trauma in the primary dentition did not increase root resorption frequency in permanent incisors.

\section{Acknowledgements}

The research was funded by Coordenação de Aperfeiçoamento Pessoal de Nível Superior - CAPES.

The authors would like to thank Billy Hanes, Translingual Comunicações Ltda.

9. Rasmusson CG, Koch G. Assessment of traumatic injuries to primary teeth in general practise and specialized paediatric dentistry. Dent Traumatol. 2010 Apr;26(2):129-32.

10. Amorim LF, Costa LR, Estrela C. Retrospective study of traumatic dental injuries in primary teeth in a Brazilian specialized pediatric practice. Dent Traumatol. 2011 Oct;27(5):368-73.

11. Marodi L. Innate cellular immune responses in newborns. Clin Immunol. 2006 Feb-Mar;118(2-3):137-44.

12. Burl S, Townend J, Njie-Jobe J, Cox M, Adetifa UJ, Touray E, et al. Age-dependent maturation of toll-like receptor-mediated cytokine responses in Gambian infants. PLoS One. 2011 Apr;6(4):e18185.

13. Tulic MK, Andrews D, Crook ML, Charles A, Tourigny MR, Moqbel R, et al. Changes in thymic regulatory t-cell maturation from birth to puberty: Differences in atopic children. J Allergy Clin Immunol. 2012 Jan;129(1):199-206.

14. Martin R, Nauta AJ, Ben Amor K, Knippels LM, Knol J, Garssen J. Early life: Gut microbiota and immune development in infancy. Benef Microbes. 2010 Nov;1(4):367-82.

15. Dietert RR, Piepenbrink MS. The managed immune system: Protecting the womb to delay the tomb. Hum Exp Toxicol. 2008 Feb;27(2):129-34.

16. Snell-Bergeon JK, Smith J, Dong F, Baron AE, Barriga K, Norris JM, et al. Early childhood infections and the risk of islet autoimmunity: The diabetes autoimmunity study in the young (daisy). Diabetes Care. 2012 Dec;35(12):2553-8. 
17. Sameshima GT, Sinclair PM. Predicting and preventing root resorption: Part II. Treatment factors. Am J Orthod Dentofacial Orthop. 2001 May;119(5):511-5.

18. Sameshima GT, Sinclair PM. Predicting and preventing root resorption: Part I. Diagnostic factors. Am J Orthod Dentofacial Orthop. 2001 May;119(5):505-10.

19. Roskamp L, Westphalen VP, Carneiro E, Fariniuk LF, Silva Neto UX. The positive influence of atopy on the prognosis of avulsed and replanted teeth despite differences in post-trauma management. J Endod. 2011 Apr;37(4):463-5.

20. Owman-Moll P, Kurol J. Root resorption after orthodontic treatment in high- and low-risk patients: Analysis of allergy as a possible predisposing factor. Eur J Orthod. 2000 Dec;22(6):657-63.

21. Malmgren B, Andreasen JO, Flores MT, Robertson A, DiAngelis AJ, Andersson L, et al. International association of dental traumatology guidelines for the management of traumatic dental injuries: 3. Injuries in the primary dentition. Dent Traumatol. 2012 Jun;28(3):174-82.

22. Balducci L, Ramachandran A, Hao J, Narayanan K, Evans C, George A. Biological markers for evaluation of root resorption. Arch Oral Biol. 2007 Mar;52(30:203-8.

23. Jágr M, Eckhardt A, Pataridis S, Miksik I. Comprehensive proteomic analysis of human dentin. Eur J Oral Sci. 2012 Aug;120(4):259-68. 